\title{
Numerical Approximation of Mindlin-Reissner Plates
}

\author{
By F. Brezzi and M. Fortin \\ Dedicated to Professor Joachim A. Nitsche on the \\ occasion of his sixtieth birthday
}

\begin{abstract}
We consider a finite element approximation of the so-called Mindlin-Reissner formulation for moderately thick elastic plates. We show that stability and optimal error bounds hold independently of the value of the thickness.
\end{abstract}

1. Introduction. The so-called Mindlin-Reissner model for moderately thin plates is often used by engineers in connection with plate and shell problems. It is well known that many numerical schemes for this model are satisfactory only when the thickness parameter $t$ is "not too small". For a very small $t$, some bad behavior (such as the "locking" phenomenon) might occur. Here we present a method which is uniformly good as $t$ goes to zero, and we prove optimal error estimates for transversal displacement, rotations and shear stresses, with constants independent of $t$.

An outline of the paper is as follows. In Section 2 we recall the Mindlin-Reissner formulation and we construct a "model sequence" of problems $\left\{\mathscr{P}_{t}\right\}_{t>0}$, where $t$ is the thickness of the plate. In Section 3 we describe our discretization procedure and we prove optimal error bounds.

A different kind of discretization of this Mindlin-Reissner model is discussed in [4]. For the one-dimensional case, a deep analysis is done in [1]. For some recent survey on other techniques used in the engineering literature, see [5], [8].

2. The Mindlin-Reissner Model. Let $\Omega$ be, for the sake of simplicity, a convex polygon in $\mathbf{R}^{2}$. The plate will occupy, in the undeformed configuration, the region $\Omega \times]-t, t\left[(t=\right.$ thickness, $>0)$. If $\left(0,0, f_{3}\right)$ is the (vertical) load per unit volume acting on the plate, the Mindlin-Reissner model can be written as

$$
\underset{\underline{\beta}, w}{\operatorname{Minimize}} \Pi:=\frac{t^{3}}{2} a(\underline{\beta}, \underline{\beta})+\frac{\lambda t}{2}\|\underline{\nabla} w-\underline{\beta}\|_{0, \Omega}^{2}-\int_{\Omega \times]-t, t[} f_{3} w d x d y d z,
$$

where $\underline{\beta}$ and $w$ are functions of $(x, y) \in \Omega$ and

$$
\begin{aligned}
a(\underline{\beta}, \underline{\eta}):=\frac{E}{12\left(1-\nu^{2}\right)} \int_{\Omega}\{( & \left.\frac{\partial \beta_{1}}{\partial x}+\nu \frac{\partial \beta_{2}}{\partial y}\right) \frac{\partial \eta_{1}}{\partial x}+\left(\nu \frac{\partial \beta_{1}}{\partial x}+\frac{\partial \beta_{2}}{\partial y}\right) \frac{\partial \eta_{2}}{\partial y} \\
& \left.+\frac{(1-\nu)}{2}\left(\frac{\partial \beta_{1}}{\partial y}+\frac{\partial \beta_{2}}{\partial x}\right)\left(\frac{\partial \eta_{1}}{\partial y}+\frac{\partial \eta_{2}}{\partial x}\right)\right\} d x d y,
\end{aligned}
$$

Received July 26, 1985.

1980 Mathematics Subject Classification. Primary 65N30, 73K10, 73K25. 
and where $t, \nu$, and $E$ respectively denote thickness, Poisson's ratio and Young's module. One also has $\lambda=E k / 2(1+\nu)$, with $k=$ correction factor, to account for the vanishing of the stress field on the upper and lower face of the plate. See, for instance, [3] for more details.

In order to study the behavior of the discretization of $(2.1)$ for smaller and smaller $t$, we need a sequence of problems such that the corresponding solutions remain bounded. For this we assume in (2.1) that a sequence of loads $f_{3}(t)$ is given by

$$
f_{3}(t ; x, y, z)=\frac{t^{2}}{2} g(x, y)
$$

hence for any $t>0$ we consider the problem

$$
\underset{\underline{\beta}, w}{\operatorname{Minimize}} \Pi_{t}:=\frac{t^{3}}{2} a(\underline{\beta}, \underline{\beta})+\frac{\lambda t}{2}\|\underline{\nabla} w-\underline{\beta}\|_{0, \Omega}^{2}-t^{3} \int_{\Omega} g w d x d y .
$$

For the sake of simplicity, we shall consider the case of a clamped plate. This implies that the minimum in (2.4) has to be taken under the kinematic constraint $\underline{\beta}=w=0$ on $\partial \Omega$. More precisely, we set

$$
V=\left\{(\underline{\theta}, \xi) \mid \underline{\theta} \in\left(H_{0}^{1}(\Omega)\right)^{2}, \xi \in H_{0}^{1}(\Omega)\right\},
$$

and we look for $(\underline{\beta}, w) \in V$.

The following proposition holds (cf. [9], [4]).

Proposition 2.1. For every $t>0$, problem (2.4) has a unique solution $\underline{\beta}(t), w(t)$. Moreover, we have, as $t \rightarrow 0$,

$$
(\underline{\beta}(t), w(t)) \rightarrow(\underline{\beta}, w) \text { in } V,
$$

where $\underline{\beta}=\underline{\nabla} w$ and $E \Delta^{2} w=12\left(1-\nu^{2}\right) g$.

Moreover, for numerical purposes, it is also convenient to have a bound on the quantities

$$
\underline{\gamma}(t):=t^{-2}(\underline{\nabla} w(t)-\underline{\beta}(t)),
$$

related to the shear stresses. For this we introduce the space

$$
\left\{\begin{array}{l}
H_{0}(\operatorname{rot} ; \Omega):=\left\{\underline{\eta} \mid \underline{\eta} \in\left(L^{2}(\Omega)\right)^{2}, \text { rot } \underline{\eta} \in L^{2}(\Omega), \underline{\eta} \cdot \underline{\tau}=0 \text { on } \partial \Omega\right\}, \\
\|\underline{\eta}\|_{H_{0}(\operatorname{rot}: \Omega)}^{2}:=\|\underline{\eta}\|_{0, \Omega}^{2}+\|\operatorname{rot} \underline{\eta}\|_{0, \Omega}^{2}
\end{array}\right.
$$

(here $\operatorname{rot} \underline{\eta}=\left(\partial \eta_{2} / \partial x-\partial \eta_{1} / \partial y\right)$ and $\underline{\tau}=$ unit counterclockwise tangent to $\partial \Omega$ ). We also introduce

$$
\Gamma:=\left(H_{0}(\operatorname{rot} ; \Omega)\right)^{\prime} \equiv\left\{\begin{array}{l}
\left\{\underline{\eta} \mid \underline{\eta} \in H^{-1}(\Omega), \operatorname{div} \underline{\eta} \in H^{-1}(\Omega)\right\} \\
\|\underline{\eta}\|_{\Gamma}^{2}:=\|\underline{\eta}\|_{-1, \Omega}^{2}+\|\operatorname{div} \underline{\eta}\|_{-1, \Omega}^{2}
\end{array}\right.
$$

Then we have (cf. [9], [4]), denoting $\langle\cdot, \cdot\rangle$ duality between $H_{0}(\operatorname{rot} ; \Omega)$ and $\Gamma$,

Proposition 2.2. The sequence (2.5) is bounded in $\Gamma$; moreover, as $t \rightarrow 0$,

$$
\underline{\gamma}(t) \rightarrow \underline{\gamma} \text { in } \Gamma
$$

with $a(\underline{\beta}, \underline{\eta})+\langle\underline{\gamma}, \underline{\eta}\rangle=0$ for all $\underline{\eta} \in\left(H_{0}^{1}\right)^{2}$. 
Our purpose is now to find a discretization procedure for (2.1) such that, on the model sequence (2.4), the corresponding error estimates hold uniformly in $t>0$.

To do that, we first give a different formulation of (2.4). The new formulation will be better suited for our discretization scheme. For this purpose, we give a different characterization of the space $\Gamma$ defined in (2.6).

Proposition 2.3. Every element $\underline{\eta} \in \Gamma$ can be written in a unique way as

$$
\underline{\eta}=\underline{\nabla} \psi+\underline{\operatorname{rot}} p \quad\left(\underline{\operatorname{rot}} p:=\left(\frac{\partial p}{\partial y},-\frac{\partial p}{\partial x}\right)\right),
$$

with $\psi \in H_{0}^{1}(\Omega)$ and $p \in L^{2}(\Omega) / \mathbf{R}$. Moreover,

$$
\|\underline{\eta}\|_{\Gamma}^{2}=\|\psi\|_{1, \Omega}^{2}+\|p\|_{L^{2}(\Omega) / \mathbf{R}}^{2}
$$

Proof. Set $\chi:=\operatorname{div} \underline{\eta} \in H^{-1}(\Omega)$. Then $\psi$ is the unique solution of $\Delta \psi=\chi$ in $\Omega$, $\psi \in H_{0}^{1}(\Omega)$. Note that now $\operatorname{div}(\underline{\eta}-\underline{\nabla} \psi)=0$. Hence, $\underline{\eta}-\underline{\nabla} \psi=\operatorname{rot} p$, and $p$ is determined in $L^{2}(\Omega) / \mathbf{R}$ (that is, up to a constant). Then we have (2.8). The proof of (2.9) is immediate.

Remark 2.1. It must be noted that $\underline{\gamma} \in H_{0}(\operatorname{rot} ; \Omega)$ could be written as $\underline{\gamma}=\underline{\nabla} \psi$ $+\operatorname{rot} p$ with $\psi \in H_{0}^{1}$ and $p \in H^{1}(\Omega) / \overline{\mathbf{R}}$. The difference between $H_{0}(\operatorname{rot} ; \Omega)$ and $\Gamma$ can thus be understood as a matter of regularity of the $p$ component. This also explains the convergence results that follow.

Note now that problem (2.4) can be written as follows:

Find $\underline{\beta}(t), w(t), \psi(t), p(t) \in\left(H_{0}^{1}\right)^{2} \times H_{0}^{1} \times H_{0}^{1} \times H^{1} / \mathbf{R}$ such that

$$
\begin{aligned}
& a(\underline{\beta}(t), \underline{\eta})-\lambda(\underline{\nabla} \psi(t)+\underline{\operatorname{rot}} p(t), \underline{\eta})=0 \quad \forall \underline{\eta} \in\left(H_{0}^{1}\right)^{2}, \\
& \lambda(\underline{\nabla} \psi(t), \underline{\nabla} \xi)=(g, \xi) \quad \forall \xi \in H_{0}^{1}, \\
& (\underline{\nabla} w(t)-\underline{\beta}(t), \underline{\nabla} x)=t^{2}(\underline{\nabla} \psi(t), \underline{\nabla} x) \quad \forall \chi \in H_{0}^{1}, \\
& (-\underline{\beta}(t), \underline{\operatorname{rot}} q)=t^{2}(\underline{\operatorname{rot}} p(t), \underline{\operatorname{rot}} q) \quad \forall q \in H^{1} / \mathbf{R} .
\end{aligned}
$$

Note that Eqs. (2.12), (2.13) are equivalent to

$$
\underline{\nabla} w(t)-\underline{\beta}(t)=t^{2}(\underline{\nabla} \psi(t)+\underline{\operatorname{rot}} p(t)),
$$

so that, using (2.14), Eqs. (2.10), (2.11) imply

$$
a(\underline{\beta}(t), \underline{\eta})+\lambda t^{-2}(\underline{\nabla} w(t)-\underline{\beta}(t), \underline{\nabla} \xi-\underline{\eta})=(g, \xi) \quad \forall \xi \in H_{0}^{1} \forall \underline{\eta} \in\left(H_{0}^{1}\right)^{2} .
$$

Now the equivalence between (2.4) and (2.10)-(2.13) is clear, since (2.15) is just the variational formulation of (2.4). It follows from Proposition 2.2 that, in particular, $\psi(t)$ will be bounded in $H_{0}^{1}$ and $p(t)$ will be bounded in $L^{2}(\Omega) / \mathbf{R}$ as $t \rightarrow 0$.

We point out that Eqs. (2.10), (2.13) have a "more natural" ordering. More precisely, for $g$ given, say, in $L^{2}(\Omega)$, one can start by solving (2.11) first. Then joining together (2.10) and (2.13) we have

$$
\left\{\begin{array}{l}
a(\underline{\beta}(t), \underline{\eta})-\lambda(\underline{\operatorname{rot}} p(t), \underline{\eta})=\lambda(\underline{\nabla} \psi(t), \underline{\eta}) \quad \forall \underline{\eta} \in\left(H_{0}^{1}\right)^{2}, \\
-(\underline{\beta}(t), \underline{\operatorname{rot}} q)=t^{2}(\underline{\operatorname{rot}} p(t), \underline{\operatorname{rot}} q) \quad \forall q \in H^{1} / \mathbf{R} .
\end{array}\right.
$$


We remark that, by setting

$$
\underline{\eta}^{\perp}:=\left(-\eta_{2}, \eta_{1}\right)
$$

problem (2.16) can be written as

$$
\left\{\begin{array}{l}
a\left(\underline{\beta}^{\perp}(t), \underline{\eta}\right)+(p(t), \operatorname{div} \underline{\eta})=\lambda\left(\underline{\nabla} \psi(t), \underline{\eta}^{\perp}\right) \quad \forall \underline{\eta} \in\left(H_{0}^{1}\right)^{2}, \\
\left(\operatorname{div} \underline{\beta}^{\perp}(t), q\right)=t^{2}(\underline{\nabla} p(t), \underline{\nabla} q) \quad \forall q \in H^{1} / \mathbf{R}
\end{array}\right.
$$

which is very closely related to a Stokes problem with a "penalty term" $t^{2}\|\underline{\nabla} p\|_{0}^{2} / 2$. It is clear that (2.18) (and hence (2.16)) can be uniquely solved. Finally, one can deal with Eq. (2.12), which is again a standard problem in the unknown $w(t)$.

We can therefore summarize this by saying that the system $(2.10)-(2.13)$ is equivalent to two elliptic problems (in the variables $\psi(t)$ and $w(t)$ ) and one Stokes-like problem (in the variables $\underline{\beta}(t)$ and $p(t)$ ). We further point out the following a priori bound.

Proposition 2.4. If $\underline{\beta}(t), w(t), \psi(t), p(t)$ is the solution of $(2.10)-(2.13)$, we have

$$
\|\underline{\beta}(t)\|_{2}+\|w(t)\|_{2}+\|\psi(t)\|_{2}+|p(t)|_{1}+t|p(t)|_{2} \leqslant c\|g\|_{0}
$$

with $c$ independent of $t$.

Proof. The bound

$$
\|\psi(t)\|_{2} \leqslant c\|g\|_{0}
$$

is trivial from (2.11). Consider now the variables $\underline{\beta}(t)$ and $p(t)$, and introduce the auxiliary problem

$$
\left\{\begin{array}{l}
a(\underline{\tilde{\beta}}(t), \underline{\eta})-\lambda(\underline{\operatorname{rot}} \tilde{p}(t), \underline{\eta})=\lambda(\underline{\nabla} \psi(t), \underline{\eta}) \quad \forall \underline{\eta} \in\left(H_{0}^{1}\right)^{2}, \\
-(\underline{\tilde{\beta}}(t), \underline{\operatorname{rot}} q)=0 \quad \forall q \in L^{2} / \mathbf{R},
\end{array}\right.
$$

where $\tilde{\beta}(t)$ and $\tilde{p}(t)$ are sought in $\left(H_{0}^{1}\right)^{2} \times L^{2} / \mathbf{R}$. It is easy to check that (cf. Ladyzhenskaya [10] or Temam [12])

$$
\|\underline{\tilde{\beta}}(t)\|_{2}+|\tilde{p}(t)|_{1} \leqslant c|\psi(t)|_{1} \leqslant c\|g\|_{0} .
$$

Set now $\underline{\beta}^{*}(t):=\underline{\beta}(t)-\underline{\tilde{\beta}}(t)$ and $p^{*}(t):=p(t)-\tilde{p}(t)$. We have

$$
\left\{\begin{array}{l}
a\left(\underline{\beta}^{*}, \underline{\eta}\right)-\lambda\left(\underline{\operatorname{rot}} p^{*}, \underline{\eta}\right)=0 \quad \forall \underline{\eta} \in\left(H_{0}^{1}\right)^{2}, \\
-\left(\underline{\beta}^{*}, \underline{\operatorname{rot}} q\right)=t^{2}\left(\underline{\operatorname{rot}} p^{*}, \underline{\operatorname{rot}} q\right)+t^{2}(\underline{\operatorname{rot}} \tilde{p}(t), \underline{\operatorname{rot}} q) \quad \forall q \in H^{1} / \mathbf{R} .
\end{array}\right.
$$

Choose now $\underline{\eta}=\underline{\beta}^{*}$ and $q=p^{*}$ in (2.22); then

$$
a\left(\underline{\beta}^{*}, \underline{\beta}^{*}\right)+\lambda t^{2}\left|p^{*}\right|_{1}^{2}=-\lambda t^{2}\left(\underline{\operatorname{rot}} \tilde{p}(t), \underline{\operatorname{rot}} p^{*}\right) \leqslant c t^{2}\left|p^{*}\right|_{1}\|g\|_{0}
$$

where we used (2.21). This implies $\left|p^{*}\right|_{1} \leqslant c\|g\|_{0}$ which, from (2.21) again, gives

$$
|p(t)|_{1} \leqslant c\|g\|_{0} \text {. }
$$

From (2.20), (2.23) and (2.10) one has now easily

$$
\|\underline{\beta}(t)\|_{2} \leqslant c\|g\|_{0} .
$$


The other inequalities in (2.19) follow from (2.12) and (2.13), using (2.20), (2.23), (2.24).

Remark 2.2. The result (2.19) does not improve when $g$ is more regular or the domain $\Omega$ is smoother. For instance, one does not have, in general, $\|p(t)\|_{2}$ bounded uniformly in $t$, even for smooth $g$ and $\Omega$. The reason for this lies in the fact that the normal derivative of $p(t)$ vanishes at $\partial \Omega$. Since this is not true for $p(0)=\lim _{t \rightarrow 0} p(t)$, we have a boundary layer effect .

3. Discretization and Error Bounds. Let, as usual, $\left\{\mathscr{C}_{h}\right\}_{h}$ be a sequence of decompositions of $\Omega$ into triangles. For each $\mathscr{C}_{h}$ we set

$$
\begin{aligned}
\mathscr{L}_{1}^{1} & :=\left\{\phi\left|\phi \in C^{0}(\bar{\Omega}), \phi\right|_{T} \in \mathscr{P}_{1} \forall T \in \mathscr{C}_{h}\right\}, \\
\dot{L}_{1}^{1} & :=\mathscr{L}_{1}^{1} \cap H_{0}^{1}(\Omega), \\
B_{3} & :=\left\{\phi\left|\phi \in C^{0}(\bar{\Omega}), \phi\right|_{T} \in \mathscr{P}_{3} \text { and }\left.\phi\right|_{\partial T}=0 \forall T \in \mathscr{C}_{h}\right\} .
\end{aligned}
$$

Note that $\mathscr{L}_{1}^{1}$ and $\dot{\mathscr{L}}_{1}^{1}$ are usual spaces of piecewise linear functions, while $B_{3}$ consists of cubic bubble functions. We define now

$$
H_{h}:=\left(\dot{\mathscr{L}}_{1}^{1} \oplus B_{3}\right)^{2} ; \quad W_{h}:=\dot{\mathscr{L}}_{1}^{1} ; \quad \Gamma_{h}:=\left(\underline{\nabla} \dot{\mathscr{L}}_{1}^{1}\right) \oplus\left(\underline{\operatorname{rot}} \mathscr{L}_{1}^{1}\right) .
$$

According to the formulation (2.10)-(2.13), we can now write the discretized problem as follows:

Find $\underline{\beta}_{h}(t), w_{h}(t), \psi_{h}(t), p_{h}(t) \in H_{h} \times W_{h} \times \dot{\mathscr{L}}_{1}^{1} \times \dot{\mathscr{L}}_{1}^{1}$ such that

$$
\begin{aligned}
& \lambda\left(\underline{\nabla}_{h}(t), \underline{\nabla} \xi\right)=(g, \xi) \quad \forall \xi \in \dot{\mathscr{L}}_{1}^{1}, \\
& a\left(\underline{\beta}_{h}(t), \underline{\eta}\right)-\lambda\left(\underline{\operatorname{rot}} p_{h}(t), \underline{\eta}\right)=\lambda\left(\underline{\nabla} \psi_{h}(t), \underline{\eta}\right) \quad \forall \underline{\eta} \in H_{h}, \\
& -\left(\underline{\beta}_{h}(t), \underline{\operatorname{rot}} q\right)=t^{2}\left(\underline{\operatorname{rot}} p_{h}(t), \underline{\operatorname{rot}} q\right) \quad \forall q \in \mathscr{L}_{1}^{1}, \\
& \left(\underline{\nabla} w_{h}(t), \underline{\nabla} \chi\right)=\left(\underline{\beta}_{h}(t)+t^{2} \underline{\nabla} \psi_{h}(t), \underline{\nabla} x\right) \quad \forall \chi \in W_{h} .
\end{aligned}
$$

It is clear that (3.1) has a unique solution. Moreover, we have by standard arguments (cf. [11], [7])

$$
\left\|\psi_{h}(t)-\psi(t)\right\|_{1} \leqslant c h\|g\|_{0} .
$$

Let us consider now problem (3.2), (3.3). Keeping the analogy with the Stokes problem (see (2.18)), we see that the choice of the spaces $H_{h}$ and $\mathscr{L}_{1}^{1}$ corresponds to the use of the MINI element of [2]. In particular, we easily obtain from [2] that

$$
\operatorname{Inf}_{q \in \mathscr{L}_{1}^{1}} \operatorname{Sup}_{\underline{\eta} \in H_{h}} \frac{(\underline{\eta}, \underline{\operatorname{rot}} q)}{\|\underline{\eta}\|_{1}\|q\|_{L^{2}(\Omega) / \mathbf{R}}} \geqslant c>0,
$$

with $c$ independent of $h$. We now want to estimate the difference between $(\underline{\beta}(t), p(t))$ and $\left(\underline{\beta}_{h}(t), p_{h}(t)\right)$. We have first

$$
\begin{aligned}
&\left\|\underline{\beta}-\underline{\beta}_{h}\right\|_{1}^{2}+\lambda t^{2}\left\|\underline{\operatorname{rot}}\left(p-p_{h}\right)\right\|_{0}^{2} \\
& \leqslant a\left(\underline{\beta}-\underline{\beta}_{h}, \underline{\beta}-\underline{\beta}_{h}\right)+\lambda t^{2}\left(\underline{\operatorname{rot}}\left(p-p_{h}\right), \underline{\operatorname{rot}}\left(p-p_{h}\right)\right) \\
&= {\left[a\left(\underline{\beta}-\underline{\beta}_{h}, \underline{\beta}-\underline{\eta}\right)+\lambda t^{2}\left(\underline{\operatorname{rot}}\left(p-p_{h}\right), \underline{\operatorname{rot}}(p-q)\right)\right] } \\
&+\left[a\left(\underline{\beta}-\underline{\beta}_{h}, \underline{\eta}-\underline{\beta}_{h}\right)+\lambda t^{2}\left(\underline{\operatorname{rot}}\left(p-p_{h}\right), \underline{\operatorname{rot}}\left(q-p_{h}\right)\right)\right]=\mathrm{I}+\mathrm{II}
\end{aligned}
$$


for all $\underline{\eta} \in H_{h}$ and $q \in \mathscr{L}_{1}^{1}$. Then,

$$
\begin{aligned}
& a\left(\underline{\beta}-\underline{\beta}_{h}, \underline{\eta}-\underline{\beta}_{h}\right)=\lambda\left\{\left(\underline{\operatorname{rot}}\left(p-p_{h}\right), \underline{\eta}-\underline{\beta}_{h}\right)+\left(\underline{\nabla}\left(\psi-\psi_{h}\right), \underline{\eta}-\underline{\beta}_{h}\right)\right\}, \\
& \lambda t^{2}\left(\underline{\operatorname{rot}}\left(p-p_{h}\right), \underline{\operatorname{rot}}\left(q-p_{h}\right)\right)=\lambda\left(\underline{\beta}_{h}-\underline{\beta}, \underline{\operatorname{rot}}\left(q-p_{h}\right)\right),
\end{aligned}
$$

so that

$$
\begin{aligned}
& \mathrm{II}=\lambda\left\{\left(\underline{\nabla}\left(\psi-\psi_{h}\right), \underline{\eta}-\underline{\beta}_{h}\right)+\left(\underline{\operatorname{rot}}\left(p-p_{h}\right), \underline{\eta}-\underline{\beta}\right)\right. \\
& \left.+\left(\underline{\beta}_{h}-\underline{\beta}, \underline{\operatorname{rot}}\left(q-p_{h}\right)\right)\right\} \text {. }
\end{aligned}
$$

Choosing for $\underline{\eta}$ and $q$ the best approximations of $\underline{\beta}(t)$ and $p(t)$ in $H_{h}$ and $\mathscr{L}_{1}^{1}$, respectively, we get from (3.7), (3.8) and (3.5)

$$
\begin{aligned}
\| \underline{\beta} & -\underline{\beta}_{h} \|_{1}^{2}+\lambda t^{2}\left|p-p_{h}\right|_{1}^{2} \\
& \leqslant \operatorname{ch}\left\{\left\|\underline{\beta}-\underline{\beta}_{h}\right\|_{1}+\lambda t\left|p-p_{h}\right|_{1}+\left\|q-p_{h}\right\|_{L^{2}(\Omega) / \mathbf{R}}+h\right\} \cdot\|g\|_{0},
\end{aligned}
$$

where we also made use of (2.19).

On the other hand, from (3.6) we have

$$
\begin{aligned}
\| p_{h} & -q \|_{L^{2}(\Omega) / \mathbf{R}} \leqslant \frac{1}{c} \operatorname{Sup}_{\underline{\eta} \in H_{h}} \frac{\left(\underline{\eta}, \underline{\operatorname{rot}}\left(p_{h}-q\right)\right)}{\|\underline{\eta}\|_{1}} \\
& \leqslant \frac{1}{c} \operatorname{Sup}_{\underline{\eta} \in H_{h}} \frac{\left(\underline{\eta}, \underline{\operatorname{rot}}\left(p_{h}-p\right)\right)}{\|\underline{\eta}\|_{1}}+\|p-q\|_{L^{2}(\Omega) / \mathbf{R}} \\
& \leqslant \frac{1}{c} \operatorname{Sup}_{\underline{\eta} \in H_{h}}\left\{\frac{1}{\lambda} a\left(\underline{\beta}_{h}-\underline{\beta}, \underline{\eta}\right)+\left(\underline{\nabla}\left(\psi_{h}-\psi\right), \underline{\eta}\right)\right\} /\|\underline{\eta}\|_{1}+h|p|_{1} \\
& \leqslant \text { const }\left\{\left\|\underline{\beta}-\underline{\beta}_{h}\right\|_{1}+h\|g\|_{0}\right\},
\end{aligned}
$$

which, inserted into (3.9), gives

(3.11) $\left\|\underline{\beta}-\underline{\beta}_{h}\right\|_{1}^{2}+\lambda t^{2}\left|p-p_{h}\right|_{1}^{2} \leqslant \operatorname{ch}\left\{\left\|\underline{\beta}-\underline{\beta}_{h}\right\|_{1}+\lambda t\left|p-p_{h}\right|_{1}+h\right\} \cdot\|g\|_{0}$.

This implies

$$
\left\|\underline{\beta}-\underline{\beta}_{h}\right\|_{1}+t\left|p-p_{h}\right|_{1} \leqslant c h\|g\|_{0} .
$$

In turn, (3.12), together with (3.10), yields

$$
\left\|p-p_{h}\right\|_{L^{2}(\Omega) / \mathbf{R}} \leqslant c h\|g\|_{0} .
$$

Finally, from (2.12), (3.4), (3.5) and (3.12) we obtain

$$
\left\|w-w_{h}\right\|_{1} \leqslant c h\|g\|_{0}
$$

We conclude with the following theorem.

THEOREM 3.1. Let $\underline{\beta}(t), w(t), \psi(t), p(t)$ and $\underline{\beta}_{h}(t), w_{h}(t), \psi_{h}(t), p_{h}(t)$ be the solutions of (2.10)-(2.13) and (3.1)-(3.4), respectively. Then we have

$$
\begin{array}{r}
\left\|\underline{\beta}(t)-\underline{\beta}_{h}(t)\right\|_{1}+\left\|w(t)-w_{h}(t)\right\|_{1}+\left\|\psi(t)-\psi_{h}(t)\right\|_{1} \\
+t\left|p(t)-p_{h}(t)\right|_{1}+\left\|p(t)-p_{h}(t)\right\|_{L^{2} / \mathbf{R}} \leqslant c h\|g\|_{0},
\end{array}
$$

with $c$ independent of $h$ and $t$. 
Remark 3.1. The use of higher-order schemes in order to improve the power of $h$ in (3.14) is not clearly advantageous, because of the boundary-layer effect (see Remark 2.1) and the fact that $\|\underline{\beta}(t)\|_{3}$ may become unbounded when $t \rightarrow 0$.

Remark 3.2. It is also possible to transform Eq. (2.18) by the introduction of a mixed method for the treatment of the term $t^{2} \Delta p(t)$. More precisely, one could solve the problem:

Find $(\underline{\beta}, p, \underline{\alpha}) \in\left(H_{0}^{1}(\Omega)\right)^{2} \times L^{2}(\Omega) \times H_{0}(\operatorname{rot} ; \Omega)$ such that

$$
\begin{aligned}
& a(\underline{\beta}, \underline{\eta})+(p, \operatorname{rot} \underline{\eta})=\lambda(\underline{\nabla} \psi, \underline{\eta}), \quad \forall \underline{\eta} \in\left(H_{0}^{1}(\Omega)\right)^{2}, \\
& (\operatorname{rot} \underline{\beta}, q)=t(\operatorname{rot} \underline{\alpha}, q), \quad \forall q \in L^{2}(\Omega), \\
& t(p, \operatorname{rot} \underline{\delta})=(\underline{\alpha}, \underline{\delta}), \quad \forall \underline{\delta} \in H_{0}(\operatorname{rot} ; \Omega) .
\end{aligned}
$$

Equations (3.16)-(3.17) are a well-known weak form of the Neumann problem

$$
\left\{\begin{array}{l}
t^{2} \Delta p=\operatorname{rot} \underline{\beta} \\
\frac{\partial p}{\partial n}=0
\end{array}\right.
$$

for which successful discretizations have been developed (cf. [6]). This weaker formulation can be expected to behave better with respect to boundary-layer effects. Moreover, the limit problem for $t=0$ becomes a standard "Stokes" problem, for which very good approximations are known, using discontinuous fields for the discrete pressure. Formulation (3.15)-(3.17) also suggests that, once $p$ is computed (for $t=0$ ), an approximation $\underline{\alpha}$ of $\operatorname{rot} p$ (which is the physically interesting variable) can be obtained a posteriori by solving

$$
(\underline{\alpha}, \underline{\delta})=(p, \operatorname{rot} \underline{\delta}) ; \quad \forall \underline{\delta} \in H(\operatorname{rot} ; \Omega) ; \underline{\alpha} \in H(\operatorname{rot} ; \Omega) \text {. }
$$

Dipartimento di Meccanica Strutturale dell' Università di Pavia e Istituto di Analisi Numerica del C.N.R.

27100 Pavia, Italy

Département de Mathématiques

Université Laval

Laval, Québec, Canada G1K 7P4

1. D. N. ARNOLD, "Discretization by finite elements of a model parameter dependent problem," Numer. Math., v. 37, 1981, pp. 405-421.

2. D. N. ARnold, F. Brezzi \& M. Fortin, "A stable finite element for the Stokes equations," Calcolo, v. 21, 1984, pp. 337-344.

3. K. J. Bathe, Finite Element Procedures in Engineering Analvisis. Prentice-Hall, Englewood Cliffs. N.J., 1982.

4. K. J. Bathe \& F. Brezzi, "On the convergence of a four-node plate bending element based on Mindlin/Reissner plate theory and a mixed interpolation," in MAFELAP $V$ (J. R. Whiteman, ed.), Academic Press, London, 1985, pp. 491-503.

5. K. J. BAthe \& E. N. Dvorkin, A Formulation of General Shell Elements-The Use of Mixed Interpolation of Tensorial Components, Proc. Conf. Numerical Methods in Engineering: Theory and Applications (Swansea, Jan. 1985). (To appear.) 
6. F. BREZZI \& M. ForTin, Book in preparation.

7. P. G. Ciarlet, The Finite Element Method for Elliptic Problems, North-Holland, Amsterdam, 1978.

8. M. A. Crisfield, “A quadratic Mindlin element using shear constraints," Comput. \& Structures, v. 18 , no. 5,1984 , pp. 833-852.

9. P. DestuYnder, Thèse d'état, Université P. et M. Curie, Paris, 1980.

10. O. A. LADYZhenSKaya, The Mathematical Theory of Viscous Incompressible Flows, Gordon and Breach, New York, 1963.

11. G. Strang \& G. Fix, An Analysis of the Finite Element Method, Prentice-Hall, Englewood Cliffs, N. J., 1973.

12. R. Temaм, Navier-Stokes Equations, North-Holland, Amsterdam, 1978. 